\title{
L'INTENSITE INTERROGATIVE-EXCLAMATIVE COMME SIGNIFIANT: "LA JEUNE PARQUE"
}

\author{
MIGUELINA SOIFER
}

Les interprétations de "La Jeune Parque" constituent un matériel volumineux dans la bibliographie valérienne, ceci s'explique pleinement si l'on considère la densité de l'expression chez Valéry et la configuration simbolico-philosophique de son univers imaginatif; d'ailleurs "La Jeune Parque" a déjà été qualifiée comme "un des poèmes les plus obscurs de la littérature française"(1). La plupart des interprétations - la plus célèbre étant "La Jeune Parque commentée par Alain" - partent de l'idée essentielle selon laquelle le poème décrit "la transformation d'une conscience pendant la durée d'une nuit" $(2)$; cependant ni même les allusions du poète à son oeuvre, que l'on trouve dans des préfaces, des conférences ou des enquêtes, suffisent à dissiper l'ambigüité que revêt le poème, car elle est inhérente au style. En formulant notre interpretation du poème(3), nous nous nous sommes arrêtés sur un élément du style: le dynamisme interrogatif. exclamatif, que nous définissons comme "l'intensité affective déterminée par la fréquence des interrogations et des exclamations dans le poème". Interrogations et exclamations naissent d'un débordemment affectif dans le langage; ceci explique que la Stylistique inclut, entre les "figures de passion", les figures exclamatives (entre celles-ci l'exclamation proprement dite: "c'est l'explosion spontannée de l'âme, le cri du coeur", dit Suberville(4), aussi bien que les figures interrogatives (et l'interrogation proprement dite "c'est une demande faite impérieusement pour insister sur une idée").

Robert Le Bidois(5), analysant la motivation de la structure interrogative, lui associe une tension portant sur le désir de savoir et, en même temps, sur l'incertitude quant à la réponse. Quant à l'exclamation, c'est "un cri qui exprime un sentiment vif ou passionné" (page 73). L'affectivité interrogative-exclamative, note Le Bidois, in-

(1) Edmée de La Rochefoucauld, "Paul Valéry". Editorial Columbia, Buenos Aires, 1961.

(2) Collection Littéraire Lagarde \& Michard, "XXe. Siècle". Ed. B.rdas, p. 309.

(3) Dans l'éfude (en préparaticn) sur l'expression mystique dans la poésie de Valéry.

(4) Suberville, Jean, "Théorie de l'Art et des Genres Littéraires", Editions de l'Ecole, Paris, 1961.

(5) Le Bidois, Robert. "L'Inversion du Sujet dans la Prose Contemporaine". Editions D'Artrey, Paris, 1952. 
cide sur l'ordre linguistique et sur le plan phonétique, puisqu'il y a une mélodie interrogative et une mélodie exclamative nettement différenciées.

Nous observons que la fréquence des points de l'interrogation ef de l'exclamation n'est pas uniforme dans le poème; elle varie, augmentant dans certains passages, diminuant dans d'autres, allant jusqu'à disparaitre quelque fois: une telle variation constitue en elle-même un "langage" (un code), un contenu informatif(6) qui est aussi une autre façon de pénétrer le texte: un signifiant(7). En associant aux unités thématiques qui composent un poème les dégrés d'intensité affective que nous considerons ici, nous obtiendrons une nouvelle donnée d'information relative au contenu du poème.

Selon notre interprétation, "La Jeune Parque" exprime dans ses 512 vers, une succession de mouvements animiques déterminés par une impulsion essentielle du moi: le désir de communication avec une Présence transcendente.

Cette impulssion détermine les autres mouvements ou réactions qui sont:

1) La pleine communication de l'extase; 2) révolte devant l'impossibilité d'atteindre la Présence; 3) évasion dans la vie sensible; 4) évasion dans son propre monde intérieur; 5) évasion dans le monde naturel environnant; 6) attente de l'extase.

A chacun de ces contenus qui s'articulent tout au long du poème, correspond une quantité ou fréquence de points d'interrogation ou d'exclamation, fréquence que nous appellerons, par ordre croissant: intensité minimale, basse, moyenne, haute. Le poème est ainsi "réduit" au schemas ou succession thématico-dynamique suivante:

Vers 1-49: éveil de la protagoniste à la révelation. En s'interrogeant sur l'irruption du surnaturel, elle décrit une Présence faiblement.

(6) Ce point de vue appartient à la Théorie de l'Information de C. Shannon. Dans l'interprétation probabilistique, ces signes (!et?) nous donneraient une information "technique" indépendante de l'information "sémantique". C'est par les études de fréquence au niveau informationnel qu'on est arrivé au concept d'entropie. Cf. Shannon, C. E. and Warren, W "The Mathematical Théory of Communication". Hegenberg, 1. e Berman, M.S. "Língua e Teoria da Informação" in "O Estado de São Paulo", Atualidades Cientificas, 24/XII/1967. - Berman M.S. "Entropia das Palarras da Llingua Portuguêsa". Laboratório de Processamento de Dados, I.T.A., Centro Téenico de Aeronáutica, São Paulo, 1967.

(7) Le concept de "significante", définitivement incorporé à la Stylistique a été élaboré par Damaso Alonso d partir du vocabulaire saussurien. "Significante es para nosotros, repetimos, tado to que en el habla modifica leve o grandemente nuestro sistema psíquico; lo mismo ese

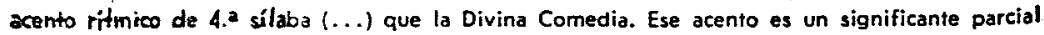
levísimo; el poema, un significante enormemente complejo. - Dámaso Alonso "Poesía Española". Ensayo de Métodos y Límites Estilisticos". Editorial Gredos, Madrid, 1950. 
insinuée. 49 vers avec 0 (?) et 3 (!): intensité moyenne de prédominance interrogative.

Vers 50-101: révolte contre l'Etre qui nie la révélation totale. Cette révolte n'est pas modulée avec effusion, mais sur un ton d'orgueil exclamativement attenué. 500 vers avec - (?) et 4 (!): intensité basse.

Vers 102-148: Evocation du temps où èlle ignorait encore la Présence, quand elle vivait heureuse une vie sensitive. Évocation sereine. 40 avers avec 0 (?) ef 4 (!) intensité basse, légèrement exclamative.

Vers 149-208. Attente des visions qui dépassent"la "pythonise". Nous observons dans ces 60 vers 3 (?) et 4 (!), équilibre entre les exclamations et les interrogations; intensité minimale.

Vers 209-324. Expression de l'extase dans l'exaltation du printemps. 135 vers avec 12 (?) ef 19 (!). Intensité haute, passage central du poème.

Vers 325 a 361 . Les "signes" s'effacent. Après l'extase, redecouverte de la nature, retour au monde ambient. 36 vers avec 0 (?) et 5 (!). Intensité basse à forme exclamative.

Vers 361-380: Nouvelle attente, "vaine attente". Intensité minimale. 19 vers avec 1 (?) et 0 (!).

Vers 381-425: Retour à l'évocation de l'extase, et retour au moi. 44 vers avec 6 (?) et 9 (!). Intensité haute.

Vers 426-464: L'extase est remmemorée; 38 vers avec 6 (?) et 5 (!). Intensité haute.

Vers 465-512: Le poème s'achève sur un mouvement de concentration intérieure. 45 vers avec 0 (?) et 5 (!). Intensité basse, prédominance exclamative.

Répresentant graphiquement l'intensité interrogative-exclamative de "La Jeune Parque", on obtient le GRAPHIQUE 1; le GRAPHIQUE 2 montre l'intensité interrogative-exclamative du poème "Le Cimetière Marin" dans lequel une unité thématique coincide avec une strophe.

Considérant qu'un texte sans points d'interrogation ni d'exclamation serait graphiquement représenté par une simple ligne horizontale, on pourra apprécier immédiatement sur ces graphiques le dynamisme latent dans les poèmes.

De semblables graphiques, construits non plus en fonction du contenu affectif, mais, par exemple, en fonction de la "mélodie" interrogative-exclamative nous donneraient le dynamisme "musical" des 
poèmes: voilà proposée une méthode et ouverte une voie pour un possible large champ de recherche.

\section{INTENSITÉ INTERROGATIVE-EXCLAMATIVE DANS "LA JEUNE PARQUE"}

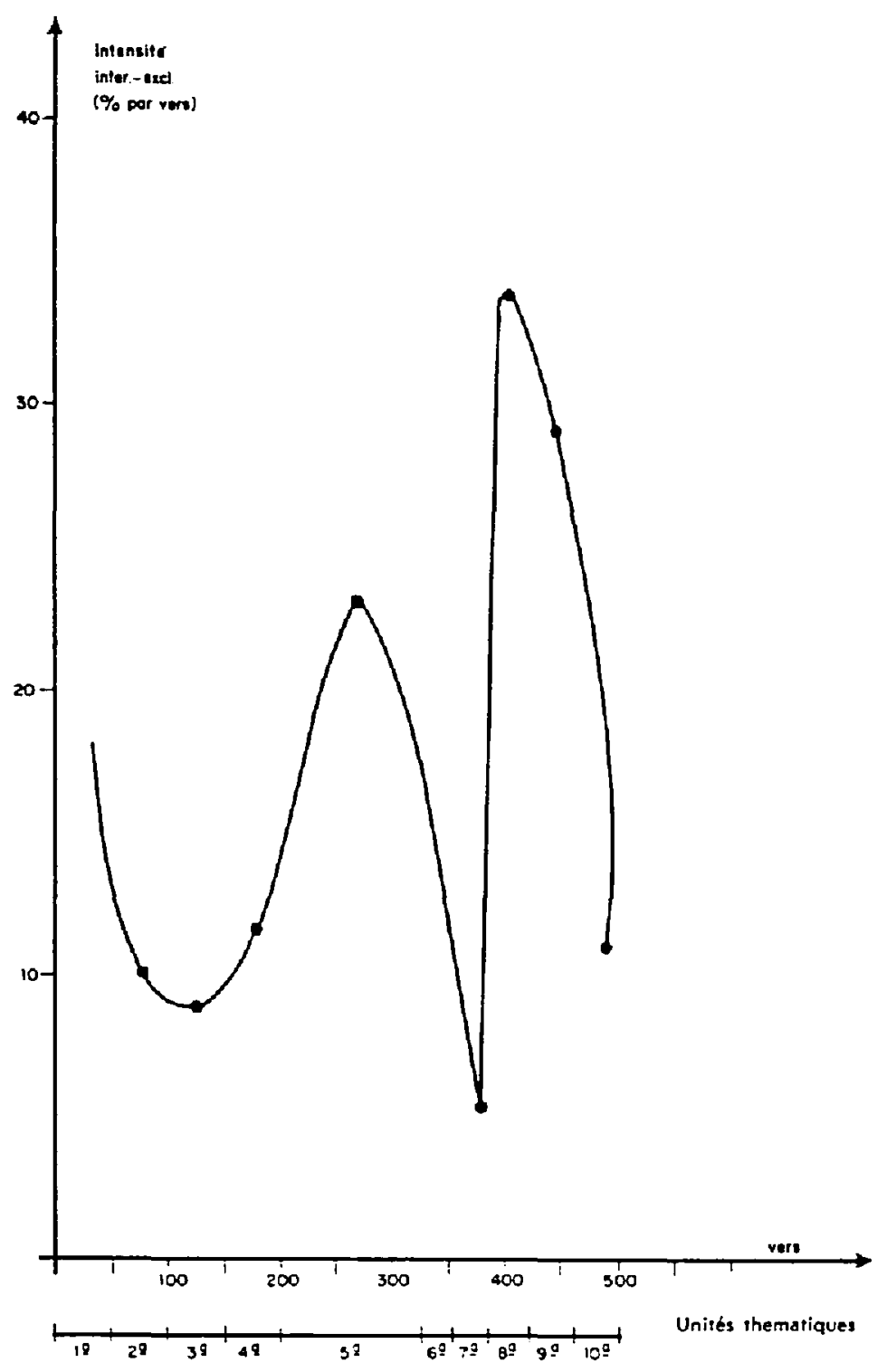

GRAPHIQUE 1 - lo courbe dintensite interrogative-exclamative est déterminée par le pourcenlage de vers svec des signes 1 ef ? (axe des ordonnés) contenus em chacune des dix unitts thématiqual (axe des abscisses). Exemple: la 2 deme. unité thématique présente $10 \%$ de ses vers signalists. 


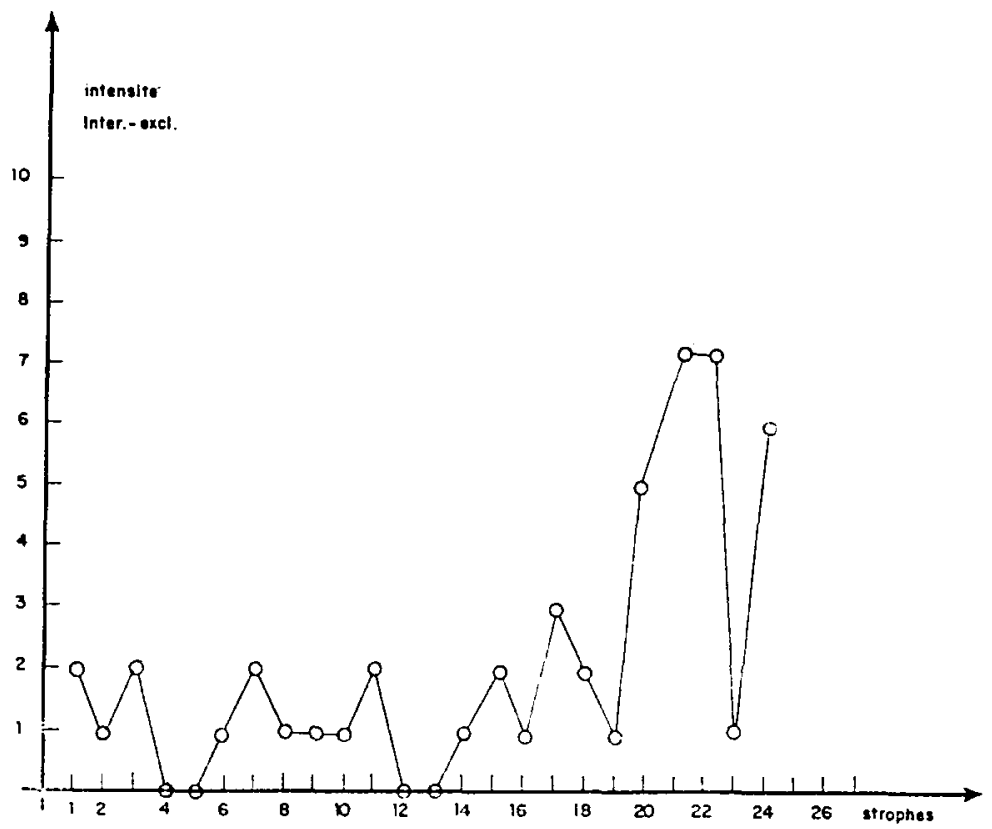

GRAPHIQUE 2 - La courbe d'intensité interrogative-exclamative est déterminée par le nombre des 1 et des ? (réprésanté dens l'axe das ordonnéss) qui apparait dens chacune des unités thématiques ou strophes (representées dans l'axe des abscisses). Exemple: La 14ème. unité thématique possède seulement 1 poins interrogatif ou exclamatif.

Nous observons que "La Jeune Parque" accuse une courbe d'intensité savamment alternée (tension moyenne - basse - basse - minimale - haute - basse - minimale - haute - haute - basse) où on distingue deux sommets (ou maxima relatifs) qui correspondent aux intensités hautes des extases, et des chutes qui correspondent aux moments d'évasion ou de concentration. Les unités d'intensités minimales semblent coincider avec les temps d'attente, tous deux précédant immédiatemment le dynamisme haut des extases. Un seul moment d'intensité moyenne: le début du poème, éveil interrogatif à la révélation.

Partant de ce reveil initial, l'impulsion mystique dans "La Jeune Parque" parviendra à l'intuition de la Présence, pour se resoudre finalement dans un mouvement de concentration intérieure:

Alors malgré moi-même il le faut, ô Soleil

que j'adore mon coeur où tu te viens connaître.

Voilà la trajectoire du mysticisme valérien, voilà l'abîme qui le sépare du mystique Saint Jean de la Croix, dont les poèmes s'achèvent dans la béatitude de la fusion. 